\title{
Unconventional Gas Resources: Economics \& Developments
}

Authors: George C. Yeung, Mohammad A. Mian, Anwar M. Beaiji, Saudi Aramco

Unconventional gas (UG) refers to natural gas resources trapped in coalbeds, shale formations and tight sand reservoirs with very low permeability (micro Darcy). UG resources, shale gas in particular, is considered as a "game changer" global gas markets. Policymakers in many countries with shale gas resources are seeking to replicate the success of shale gas in the United States. A recently published US Energy Information Administration (EIA) sponsored report shows 6,622 trillion cubic feet of technically recovered shale gas resources spread over 48 shale gas basins in 32 countries and containing almost 70 shale gas formations.

In order to explore and economically develop UG resources, geologists, completion engineers and production engineers face difficult challenges. UG resources differ significantly from conventional gas reservoirs where exploitation of these resources follows different decision gates: Exploration - Appraisal - Pilot - Full Field Development. Also, gas production declines sharply resulting in relatively low Estimated Ultimate Recovery per well (EUR/Well) and extensive drilling requirements. UG resources are continuous accumulations of natural gas covering very large geographical areas. One of the greatest challenges in UG resources exploration and development is drilling and completion, where it is necessary to perform efficient completion techniques that will maximize gas recovery and minimize drilling and completion costs. Horizontal drilling and Multistage Hydraulic Fracturing is now the common practice to develop these resources where large volumes of water are required for fracing jobs. Safe recycling or disposal of these produced fluids is necessary. Along with other challenging characteristics of UG, resources a large number of wells must be drilled to maintain an economic plateau resulting in denser well spacing which leads to the need of large surface facilities and infrastructures to tie-in these wells. These challenges contribute to the relatively higher Breakeven Gas Price (BGP) of UG resources developments. This paper provides an insight on (a) technological and logistic challenges in exploiting UG resources, (b) planning for shale gas resource exploitation, and (c) economic analysis workflow of UG development. 